\title{
Control and relation: two foundational values and their consequences*
}

\author{
ANTHONY L. SUCHMAN
}

The University of Rochester School of Medicine and Dentistry, New York, USA

\begin{abstract}
This paper explores and contrasts personal philosophies based on two different core values, control and relation, with respect to expectations, social relationships, habits of perception and interpretation, and ways of feeling grounded in the world. The paradigm of control is widespread in medicine and certain other health professions, but because it fosters unrealistic expectations, evokes fear and shame, and inhibits effective partnerships, it can actually compromise health outcomes. The paradigm of relation calls attention to interpersonal process and fosters receptivity and adaptability, thus enhancing partnership. A mature clinical approach combines these two perspectives, respecting both the benefits and limitations of reductionistic science and making room for self-organization and emergence.
\end{abstract}

Keywords: Control, relationships, values and consequences

\section{Introduction}

The deepest level determinant of partnership, in health care or anywhere else, is our internal predisposition as would-be partners: our attitudes, values and beliefs. Each of us has a personal philosophy that shapes our perceptions, interpretations and actions. Addressing questions such as why things happen, what is right and what is wrong, and why we are here, our personal philosophies give rise to our own individual ways of being in the world; they also frame our goals and expectations. In adopting a particular personal philosophy, we create the world we inhabit.

Considering its profound importance and implications, the acquisition of our personal philosophy is remarkably uncritical. This process - a gradual assimilation of attitudes and values from our families, culture, education, and life experiences - tends to be so subliminal that we may not recognize our core beliefs as beliefs at all, and instead simply accept them as 'reality'. We may not recognize that our 'truths' are in fact only 'assumptions', and that better alternatives might exist.

In this paper, we will examine one of the most fundamental values in Western culture, one that figures prominently in the personal philosophies of most health professionals: the quest for control. As we explore the control paradigm with regard to its core values, goals, patterns of social relationships, approaches to gathering and using knowledge, and sources of existential security (see Table I), we will see the limitations it imposes on the process of partnership. We will then examine an alternative paradigm based on the relation that creates

\footnotetext{
*Reproduced with permission from Suchman, A. L., Hinton-Walker, P. \& Botelho, R. J. (1998). Partnerships in healthcare: transforming relational process. New York: The University of Rochester Press.

Correspondence: Anthony L. Suchman, The University of Rochester School of Medicine and Dentistry, New York, USA.
} 
a climate more conducive to creative collaboration and the sharing of responsibility. Although we will use clinicians as exemplars throughout this discussion, the concepts are equally applicable for educators and administrators.

In making an explicit comparison of the control and relation paradigms, we can learn to perceive the values implicit in everyday interactions and to observe their consequences. Then, perhaps for the first time, we can make thoughtful choices about which paradigm to embrace as our own.

\section{The paradigm of control}

\section{Core values and goals}

The beliefs, thoughts and behaviors of the control paradigm are organized around a single core value: that the ultimate state to which one can aspire is one of perfect willfulness and predictability. What one desires happens, with no surprises; all outcomes are intended. For the clinician, the control paradigm is expressed in the questions, "What do I want to happen here?" and "What's wrong and how do I fix it?" The 'will to fix' extends well beyond the patient's immediate experience of suffering or dysfunction to encompass intermediate outcomes believed to be associated with future suffering (for example, reduction of blood pressure or glycohemoglobin levels). Thus, the paradigm of control defines the clinician's role as taking deliberate action towards an improvement in the patient's current or future experience. Personal success or failure is judged by the clinical outcome, the extent to which one's intended outcome was realized.

\section{Social relationships}

Interpersonal relationships in the control paradigm are hierarchical. At the level of the physician-patient relationship, for example, the physician, by virtue of expertise and responsibility, makes decisions on behalf of the patient, who is expected to comply. This use of power to limit the choices of others poses no ethical problem for the physician, so long as the intended outcome is the patient's improvement. Relationships on the health care team are hierarchical, as well. Differences in educational and social status, defined areas of responsibility and professional prerogatives create expectations of deference and obedience. In situations of conflict, the control paradigm predisposes the physician to focus on goals and to expect to be dominant, resulting in a win-lose negotiation style (if negotiation takes place at all).

\section{Epistemology}

The control paradigm shapes both epistemology-the way we gather and organize knowledge-and clinical reasoning. With control as the principal value, the task of intellectual inquiry is to develop detailed knowledge about causal mechanisms and how to manipulate them. The mode of analysis to produce such knowledge is reductionistic and mechanistic, and tends to move from the particular to the general; that is, it values knowledge of general principles and theories more than knowledge about individual experience (which is dismissed so often as 'anecdotal'). When applied to clinical work, this approach leads the clinician to encapsulate an episode of a patient's life experience with a single diagnostic label. Just by this act of classification, the clinician gains a degree of intellectual control and moves from a state of uncertainty towards one of mastery. 
Treatment is chosen more on the basis of generalized knowledge about the diagnosed disease than about the particularity of the patient's experience. So in both making a diagnosis and planning a treatment, what is unique and particular about an individual patient is less valued and therefore gets less attention than the abstracted characteristics of the 'case'.

Rational thought, in its orderliness and reproducibility, is most consistent with the philosophy of control, and is therefore valued above all other forms of mental experience. Personal meanings, interpretations and emotions, being 'irrational', are deemed inferior, hence less worthy of attention. Objective experience, as the substrate of rational thought, is deemed more relevant-even more real-than subjective experience. In focusing on their own objective experience and suppressing their subjectivity, clinicians take the role of detached observer, distanced from the object of observation and affectively neutral.

\section{Existential security}

The core of the control paradigm, the source of its compelling power, lies deeper than cognition and behavior, at the level of existential security. Questions at this deepest level include: what is the nature of the world? What stance does one take? Where does one find one's grounding? At the heart of the quest for control is fear, a perception of the world as lacking any intrinsic order and of danger lurking in the chaos. One's grounding, or source of security, is in predictability - the order one imposes on the world through collective and individual efforts. Collective efforts include rules of interpersonal engagement and knowledge structures as described above. Individual efforts include staying within prescribed boundaries, obeying rules and resisting the 'irrational'. Relaxing one's vigilance and losing control of the details of one's life opens the doors to chaos and to bad things happening. The worst thing of all is something bad happening that could have been prevented but was not. The clinician's existential stance in the control paradigm - his basic way of being in the world - is one of mastery: of the instrumental knowledge of medicine and of other people; in metaphorical terms, a stance of holding tight. We may trust others to a point, but we trust nothing and no one as much as we trust ourselves. Therefore, mastery, vigilance and self-sufficiency are the ultimate source of existential security, the best way to keep the fear at bay.

\section{Limitations of the control paradigm}

That the control paradigm has channeled human energy towards many remarkable technological accomplishments is undeniable. However, as a philosophy for guiding human interactions and for providing existential grounding, it has serious limitations. These are especially significant in medicine, where technology and human experience intersect with particular drama and salience.

The control paradigm interferes in a variety of ways with the formation of relationships, the medium of partnership. The instrumental, reductionistic approach of the control paradigm and the valuation of the abstract general over the particular leads to an inadvertent depersonalization of others. Patients, students and subordinates become objects to be controlled. The hierarchy of the control paradigm blocks the free flow of communication that is necessary to achieve synergistic interaction. It promotes expectations for those lower down in the hierarchy that the people higher up will be able to control things, leading them to expect little of themselves, thus promoting passivity. There is little opportunity for the 
kind of mutual goal setting that enhances motivation and commitment. The full power of the team goes untapped.

At the level of thinking and action, the control paradigm encourages an appealing yet utterly unrealistic fantasy of personal control, focusing on the individual as the primary locus of agency. It interferes with the perception of systems and the recognition of emergent phenomena-synergistic processes which are effectively and spontaneously orchestrated through complex interactions among individuals.

The control paradigm accounts for a number of common problems in clinical practice. For example, consider the way doctors become angry with patients who do not take their medicines. The physicians' sense of personal success and mastery depends upon the clinical outcome; the patients' 'noncompliance' jeopardizes the outcome and thus threatens the physicians' well being. Consequently, the physicians become angry, as if it was they and not the patients who had to bear the ultimate consequences. Another common problem is the abandonment of patients with chronic or terminal illness. In these settings, clinicians are predisposed to experience their inability to cure as failure. Not surprisingly, they tend to withdraw, or they may cling to inflated expectations of success and undertake excessive treatment, frequently substituting their own goals for those of their patients.

Ultimately, the control paradigm fails as source of existential security. It is built on two false premises. First, it is not possible to implement perfectly what one wills, and second, even if it were possible to do so, the outcome would remain unpredictable. We know from chaos theory (enshrined in conventional wisdom as the Law of Unintended Consequences) that predictability pertains only within very narrowly defined situations, and is generally a poor construct for modeling natural and social phenomena (Gleick, 1987). Complexity prevails. Expectations of control are created that cannot be fulfilled, resulting in feelings of disillusionment, anger and personal failure (known also as 'burnout'). The control model ultimately must prove to be an inadequate map; at some point its discrepancies with the real world must cause it to create more problems than it solves.

\section{The paradigm of relation}

\section{Core values and goals}

An alternative paradigm organized around the core value of relation has the potential to avoid many of the problems of the control paradigm (Toulmin, 1982). ${ }^{1}$ In the relation paradigm, the most valued state to which one aspires is one of connection and belonging. In this state, one has a feeling of being part of a larger whole - a team, a learning group, a dance troupe, a community, even the world itself. One's individual actions seem spontaneously integrated with those of others to a remarkable degree, contributing to the evolution of a higher order process, i.e. one at a higher system level than that of the individuals of which it is comprised. Words like 'serendipity', 'flow', 'synchronicity', 'self transcendence' and 'oneness' have been used to describe this ineffable state.

The relation paradigm holds that the best outcomes are realized by maximizing the quality of process, which in turn depends on the quality of relationships and on one's ability to be genuinely present to others and to events as they are unfolding. As often as not, the resulting outcomes will be different from, and better than, those that were originally intended. Being in a state of relation does not imply passivity. Rather, it involves active attentiveness to both inner and outer experience. One asks the question, "What's trying to happen here?" and, 
according to one's best approximation of an answer, seeks to shape others and the world while also remaining open to being shaped oneself. This balance between control and receptivity puts one in the best possible position to recognize and make use of serendipitous events.

Working within the paradigm of relation, the clinician's role is to 'be with' patients: to acknowledge their suffering and identify opportunities to relieve or prevent it, while also respecting their position as principal makers of values, meanings and decisions in their own lives. Thus, the professional is accountable not so much for the outcome as for the quality of the process: the degree to which one understands what the patient is experiencing; communicates that understanding back to the patient; helps the patient formulate goals and priorities; and applies one's expertise and commitment to the creation and implementation of a plan for realizing the patient's goals. These expectations afford the clinician more opportunity to feel successful than do the control paradigm's expectations of perfect clinical outcomes.

\section{Social relationships}

Interpersonal relationships under the relation paradigm are characterized by respect and genuineness. Martin Buber characterized such interactions as 'I-Thou', denoting a relationship between two subjects, as contrasted with 'I-it', denoting the subjectobject relationship of the control paradigm (Buber, 1958). One acknowledges the subjective experience, personal perspectives, values and aspirations of others and one's own as well. Self-awareness is critically important, as is the ability to act in congruence with one's feelings. One cannot be in a state of relation without being fully present.

The use of power is another key point of differentiation between the relation and control paradigms. The relation paradigm does not negate power or deny the very real differences in power between patients, doctors, nurses, administrators and others. Rather, it maintains that these differences do not imply differences of worth, dignity or the right to have one's perspective respected and, to the extent that one has power, one uses it to foster both the power and the accountability of others to the fullest extent possible (within the bounds of the individual's capacity and the external circumstances). In the realm of negotiations, this is expressed as seeking solutions which maximize mutual gain and strengthen mutual commitment (win-win) rather than maximizing one's own gain at the expense of the other (win-lose).

The receptivity of the relation paradigm leads one to value diversity. The unique background, perspective and opinion that each participant brings to an interaction or a team can be a resource to the group (provided it is approached respectfully), just as intra species diversity contributes to the adaptability and long-term survival of a species. However, this attitude of receptivity should not be mistaken for a 'do-your-own-thing' kind of uncritical tolerance. One cannot avoid making and acting on judgments about quality if there is to be accountability.

\section{Epistemology}

The epistemology of the relation paradigm seeks to join the instrumental, abstract knowledge of the control paradigm with a deep appreciation of context and ecology - a science of stories (Stange, Miller, Crabtree, O’Connor, \& Zyzanski, 1994). This has two key implications. First, one must recognize the existence of 'emergent' phenomena, those which arise at and higher orders of a system and depend on, yet cannot be 
explained in terms of, lower order processes. For instance, a cardiology consultation could not take place without normal synaptic function in the brain of the cardiologist, but the one cannot understand or even describe the process of making a diagnosis by analyzing neurotransmitters. 'Emergentism' can be seen as a style of analysis that moves across the hierarchy of systems in the opposite direction as reductionism, employing very different tools. Both approaches produce useful, distinct and complementary types of knowledge. Second, one must accept data regarding subjective experience as being equally important and relevant as objective data. Rational thought tends to support instrumentality; emotion, spiritual experience and intuition tend to be the substrate of relation.

When translated to clinical reasoning, this epistemology leads us to discover and value what is unique about each patient and his or her clinical situation, and not to concentrate solely on the abstractable, generalizable features. The accurate understanding of the patient's experience and communication of that understanding back to the patient is a vital therapeutic process (Suchman, Markakis, Beckman, \& Frankel 1997), and an essential part of relationship building (Cohen-Cole, 1991). Likewise, the clinician must be self-aware to be capable of relationship (Novack et al. 1997). So information about the subjective experience of the patient and clinician is important information. Rational analysis and experiential exploration both have their place. The clinician's role, far from one of detachment, is to participate and to observe both her outer (objective) and inner (subjective) experience (Engel, 1988).

One last aspect of epistemology for us to consider is the relinquishing of answers. Each time one arrives at an answer, one's desire for mastery may be satisfied, but if answers terminate inquiry, further discovery (and the revision of erroneous answers) is precluded. One needs provisional answers to undertake action; medical care, education and administration constantly demand action in the face of uncertainty. Nevertheless, one must avoid holding too tightly to answers and be willing instead to remain always in the question' (Goodrich-Dunn, 1991).

\section{Existential security}

At the core of the relation paradigm is trust. This philosophy accepts that there are sources of order, goodness and meaning beyond one's own creation. This source may be at a collective human level (as exemplified by the consistent performance advantage of wellfunctioning teams over individuals) or on an even higher order: the 'Tao', 'God', and 'Great Spirit' are but a few of the names humans have given in reference to universal sources of order and meaning. Regardless of scale, the basic existential stance in the relation paradigm is one of self-transcendence and receptivity, of letting go of control and remaining open. One's source of existential security might best be characterized as alignment: recognizing the interdependence of oneself and others in a larger order of things and learning to be part of the flow of that order.

\section{Further implications of the relation paradigm}

It is important to recognize that the relation paradigm does not negate the control paradigm; rather, it promotes balance by adding receptivity to mastery, subjective to objective, emergent to reductionist, participation to autonomy. This last pair, participation and autonomy, has been particularly problematic for practitioners in today's health care 
environment. The apparently paradoxical convergence of these two opposites can be accomplished using Arthur Koestler's conceptualization of the holon (quoted in Wilber, 1996). A holon is an entity which is a complete and autonomous whole unto itself and at the same time a part of a larger whole. A holon thus has properties of both agency (corresponding to autonomy, instrumentality and control) and communion (corresponding to participation, context and relation). Until recently, physicians practiced autonomously, making clinical and business decisions from the perspective of their individual patients and their 'cottage industry' practices, respectively. They were conscious only of their agency, their completeness in themselves as loci of decision making. Now in this time of integrated health systems, they must maintain their agency, but at the same time think at higher order system level about the care of a population and about the needs of the integrated network. The relation paradigm encourages awareness of multiple system levels and allows for coexistence, rather than mutual exclusivity, of control and relation.

Looking at personal philosophies from a perspective of adult development, we can observe that aspects of the control and relation paradigms correspond to different stages of personal growth as described in two models. In Erikson's model, differentiating oneself from the world and coming to know oneself as an independent locus of agency and control is the culmination of childhood development and arrival into adulthood (Acklin, 1986). However, as one progresses through the adult stages of intimacy versus isolation (Stage VI), generativy versus. stagnation (Stage VII) and integrity versus despair (Stage VIII), one increasingly transcends the boundaries of one's self, "[creating] new conditions for, and new avenues of experiencing, enabling a richer empathy, relatedness, and identification with life beyondthe-self" (Acklin, 1986, p. 201). These themes resonate with willfulness versus alignment, mastery versus receptivity, detachment versus participation and holding tight versus letting go.

Perry's model of adult cognitive and ethical development describes nine stages of growth progressing from Dualism ("Division of meaning into two realms-Good versus Bad, Right versus Wrong, We versus They, All that is not Success is Failure, and the like. Right Answers exist somewhere for every problem, and authorities know them") to Commitment in Relativism ("Diversity of opinion, values, and judgement derived from coherent sources, evidence, logics, systems, and patterns allowing for analysis and comparison. Some opinions may be found worthless, while there will remain matters about which reasonable people will reasonably disagree. Knowledge is qualitative, dependent on contexts." "I must be wholehearted while tentative, fight for my values yet respect others, believe my deepest values [to be] right yet be ready to learn") (Perry, 1981, p. 79). Here we find resonance with the themes of reductionism and emergence, outcomes and process, and living in the question.

Taken together, these two models describe a process of personal evolution in which one's focus moves from implementing narcissistic willfulness to seeking connectedness, and one's source of grounding shifts from the existence of Authority and external absolute Truths to the experience of self transcendence and connection.

We have been considering the control and relation paradigms in the context of an individual's personal philosophy, but these paradigms are manifest in organizational process and behavior, as well (Senge, 1990). Given the considerable influence that organizations have on the perceptions, values and behaviors of the individuals who populate them, we might look to the modification of organizational process as a powerful tool for fostering personal transformation (i.e., changing personal paradigms) (Senge, 1994; Brill \& Worth, 1997). 


\section{Conclusions}

The control paradigm limits partnership. By focusing our attention on the realization of our will, on outcomes and on objective phenomena, it predisposes us towards dominance and distracts us from making observations and taking actions that might help us cultivate relationships. It creates additional problems by setting unrealistic standards for success and cutting us off from our own subjective experience. The relation paradigm avoids these pitfalls and predisposes us towards partnership. It reminds us to attend to process and to personal experience (our own and that of others), not just to objective data. It encourages us to be perpetually skeptical of answers and their false promise of predictability, and instead to live in the question, remaining receptive even as we undertake specific and decisive courses of action. Once we are able to perceive these two philosophies and their consequences, we are then able to reflect on our own experience and to make thoughtful choices about which paradigms help us live in the most meaningful and effective manner and to move forward in our personal growth.

\section{Note}

1 Whose arguments about the concept of 'relation' as the core of an alternative to the control paradigm inspired my thinking.

\section{References}

Acklin, M. W. (1986). Adult maturational processes and the facilitating environment. $\mathcal{F}$ Religion and Health, 25, $198-206$.

Brill, P. R., \& Worth, R. (1997). The four levers of corporate change. New York: AMACOM.

Buber, M. (1958). I and thou (2nd ed.). New York: Scribner's.

Cohen-Cole, S. A. (1991). The medical interview: The three function approach. St. Louis, MO: Mosby.

Engel, G. L. (1988). How much longer must medicine's science be bound by a seventeenth century world view? In: K. L. White (Ed.) The task of medicine. Dialogue at Wickenburg (pp. 13-36). Menlo Park, CA: Henry J. Kaiser Family Foundation.

Gleick, J. (1987). Chaos: Making a new science. New York: Penguin Books.

Goodrich-Dunn, B. (1991). Walking the critical path. Common Boundary, July-August, $12-21$.

Novack, D. H., Suchman, A. L., Clark, W., Epstein, R. M., Najberg, E., \& Kaplan, C. (1997). Calibrating the physicians: Physician personal awareness and effective patient care. $\mathcal{F A M A : 2 7 8 , 5 0 2 - 5 0 9}$.

Perry, W. G. (1981). Cognitive and ethical growth: the making of meaning. In: A. Chickering (Ed.). The modern American college (pp. 76-116). San Francisco: Jossey-Bass.

Senge, P. (1990). The fifth discipline. New York: Doubleday.

Senge P. (1994). The fifth discipline fieldbook. New York: Doubleday.

Stange, K. C., Miller, W. L., Crabtree, B. F., O’Connor, P. J., \& Zyzanski, S. J. (1994). Multimethod research: Approaches for integrating qualitative and quantitative methods. Fournal of General Internal Medicine, 9, 278281.

Suchman, A. L., Markakis, K., Beckman, H. B., \& Frankel, R. (1997). A model of empathic communication in the medical interview. $\mathcal{F} A M A, 277,678-682$.

Toulmin, S. (1982). The return to cosmology: Postmodern science and the theology of nature. Berkeley, CA: University of California Press.

Wilber, K. (1996). A brief history of everything. Boston: Shambala. 


\section{Appendix}

Table I. A comparison of critical features of the paradigms of control and relation with regard to core values and goals, patterns of social relationships, approaches to gathering and using knowledge, and sources of existential security.

\begin{tabular}{|c|c|c|}
\hline & Control & Relation \\
\hline Core values and goals & willfulness; predictability & connectedness; alignment \\
\hline Primary focus & outcomes & process \\
\hline Relevant domain of experience & objective only & objective and subjective \\
\hline Epistemology & reductionistic, analytic & $\begin{array}{l}\text { reductionistic and emergent; analytic } \\
\text { and experiential }\end{array}$ \\
\hline Priority of knowledge & general $\gg$ particular & general $=$ particular \\
\hline Social structure & hierarchy & partnership \\
\hline Use of power & limit choices of others & increase choices of others \\
\hline Negotiation strategy & win-lose & win-win \\
\hline Implications for clinician's stance & detached observer; affectively neutral & participant observer; fully present \\
\hline Core affect & fear & trust \\
\hline Stance & mastery & receptivity \\
\hline Source of security & self sufficiency & alignment; interdependence \\
\hline Existential strategy & holding tight & letting go \\
\hline
\end{tabular}

